\title{
Chronic total occlusion percutaneous coronary intervention complications: prevention and management
}

\author{
Francesco Colombo, Alessandro Bernardi, Roberto Garbo \\ Department of Cardiology, San Giovanni Bosco Hospital, Turin 10154, Italy. \\ Correspondence to: Dr. Francesco Colombo, Ospedale San Giovanni Bosco - Laboratorio di Interventistica Cardiovascolare, \\ Piazza del Donatore di Sangue 3, Turin, 10154, Italy. E-mail: francesco.colombo83@gmail.com
}

\begin{abstract}
How to cite this article: Colombo F, Bernardi A, Garbo R. Chronic total occlusion percutaneous coronary intervention complications: prevention and management. Vesse/ Plus 2019;3:29. http://dx.doi.org/10.20517/2574-1209.2019.005
\end{abstract}

Received: 22 Jan 2019 First Decision: 17 Jun 2019 Revised: 18 Jun 2019 Accepted: 17 Jul 2019 Published: 9 Aug 2019

Science Editor: Boukhris Marouen Copy Editor: Jia-Jia Meng Production Editor: Jing Yu

\begin{abstract}
Percutaneous coronary intervention (PCI) of coronary chronic total occlusions (CTO) still represents a challenge in the field of interventional cardiology. Despite the rate of peri-procedural complications has decreased over the years, it remains higher than in non-CTO PCl. Coronary perforations are among the most common and serious complications. Furthermore CTO recanalization carries a risk of unique and specific complications such as donor vessel injury and equipment loss or entrapment. Other infrequent complications of non-CTO PCl such as contrast induced renal dysfunction and radiation skin injury, assume more relevance in this subset given the length and complexity of these procedures. Operators facing CTO percutaneous treatment should be aware of the potential complications and the available strategies for prevention and management, to achieve procedural success.
\end{abstract}

Keywords: Chronic total occlusion; complications; coronary perforation; donor vessel injury; equipment entrapment

\section{INTRODUCTION}

Percutaneous coronary intervention (PCI) of coronary chronic total occlusions (CTO) still represents a distinct challenge in the field of interventional cardiology. The rates of successful CTO recanalization is growing, due to numerous advances in terms of newer dedicated devices, improved techniques and extensive operators' experience. Nevertheless coronary CTO treatment continues to be a challenging issue 
Table 1. Complication incidence during CTO $\mathrm{PCI}^{[1-5,16,17]}$

\begin{tabular}{ll}
\hline Complication & Incidence (\%) \\
\hline Perforation & $2.6 \%-4.8 \%$ \\
Donor vessel Injury & $1.1 \%-1.8 \%$ \\
Equipment loss or entrapment & - \\
Contrast-induced nephropaty & $3.4 \%$ \\
Vascular access complications & $0.5 \%-1.5 \%$ \\
Radiation skin injury & $<0.01 \%$ \\
Arrythmia & $0.8 \%$ \\
Stroke & $<0.01 \%$ \\
Urgent CABG & $0.1 \%$ \\
Myocardial infarction & $2.5 \%$ \\
Death & $0.2 \%$ \\
\hline
\end{tabular}

CTO: chronic total occlusions; PCI: percutaneous coronary intervention

owing to multiple reasons including the fear for peri-procedural complications ${ }^{[1]}$. Despite the rate of periprocedural complications has decreased over the years, it still remains higher than in non-CTO PCI.

The most common and feared complications occurring during CTO PCI are perforations, donor vessel dissection and/or occlusion, equipment loss or entrapment, vascular access complications, contrast-induced nephropathy, radiation skin injury, periprocedural myocardial infarction, arrhythmias, transient ischemic attack or stroke [see Table 1]. There remains considerable variability in reported complications rates in literature due to data coming mainly from single centers experience. Nevertheless, more recently, data have been made available from multicenter registries enrolling 1000 (OPEN-CTO Registry ${ }^{[2]}$ ) and even more $\left(\right.$ ERCTO Registry $\left.^{[3]}\right)$ patients in experienced CTO PCI centers.

Aim of the following chapter is to analyze the common periprocedural complications during CTO PCI, how to prevent them and the main solution techniques.

\section{CORONARY PERFORATION}

Coronary perforations (CPs) are one of the most common and serious complications occurring during CTO PCI. They have an estimated incidence of $2.6 \%-4.8 \%^{[2-6]}$ and are associated with a 5 -fold increase in 30-day mortality. Cardiac tamponade occurs in approximately $10 \%$ of patients with a coronary perforation and could represent a life-threatening situation.

General approach: when perforation is confirmed, the following measures should be played out.

- Prompt specific perforation treatment (see section below for details).

- Fast echocardiography looking for pericardial effusion and cardiac tamponade indicators (right chambers collapse, respiratory variations of antegrade mitral flow). In this setting pericardial effusion can also be detected by fluoroscopy images, especially in postero-anterior views, as contrast staining in the pericardial space.

- Fluids infusion (saline solution, blood transfusion, colloids) or vasopressors in relation to the hemodynamic conditions.

- Pericardiocentisis when necessary. Autotransfusion should be taken into account in patients with persistent bleeding despite concerns have been raised due to the potential to induce hemolysis, coagulation abnormalities or possible infections, especially when large quantities are re-infused. However this procedure was shown to be safe and effective in a recent review including 30 consecutive patients ${ }^{[7]}$.

- Pain management and eventually sedation.

- In rare cases evaluate mechanical support of circulation (ECMO/Impella/IABP). 
- Reversal of anticoagulation with protamine is not recommendable in perforations occurring during CTO interventions since it does not solve the mechanical problem underlying this complication and, on the other hand, it can dramatically increase the risk of thrombosis until all equipment are in coronary arteries.

Specific approach: different devices and techniques can be used in relation to the perforation site.

Coronary perforation have been classified by severity according to Ellis criteria ${ }^{[8]}$. Ellis classification recognizes 3 grades of perforation severity (I, II and III): while grade I can occasionally resolve without intervention or just with reversal of anticoagulation, the grade III is usually referred to abrupt and large perforations with acute cardiac tamponade and hemodynamic instability and may cause myocardial infarction and death.

Nevertheless anatomic classification based on vessel location of perforation can be more helpful, orienting in the management approach. It distinguishes three types of CPs: main vessel (MV) perforation, distal target vessel perforation and collateral channel perforation ${ }^{[9]}$.

MV perforation occurs in either antegrade or retrograde approaches. Guidewire perforation during wire escalation or dissection and re-entry techniques are typically self-limited and rarely lead to cardiac tamponade. The risk of pericardial effusion dramatically increase when a device (i.e., microcatheter or Crossboss ${ }^{\mathrm{TM}}$ ) or a balloon is advanced along a guidewire located in the pericardial space, outside the architecture of the vessel. The first highly important step is represented by prompt balloon inflation proximally to the area of contrast extravasation. If extravasation persists despite prolonged balloon inflation, then a covered stent should be implanted. Covered stent implantation generally requires a dual catheter technique ("ping-pong") in order to minimize bleeding. While a balloon is maintained inflated through the first guiding catheter, a second catheter is advanced near the coronary ostium and covered stent is positioned proximal to the occluding balloon. Then the occluding balloon is deflated and withdrawn and the covered stent is advanced and deployed. Covered stents advancement can be difficult in tortuous and calcified vessels due to the lack of flexibility of these devices. In the same way operators must take into account that their delivery across not well prepared CTO lesions should be demanding.

Distal target vessel perforation typically represents a complication of antegrade approach, occurring after CTO crossing. It is usually due to advancement of stiff and/or highly hydrophilic wires into small branches. Dual arterial injection could help to prevent this type of complication, showing vessel anatomy beyond the distal cap of the CTO. Also in this case, prompt balloon dilatation proximally to the perforation site is the first treatment step. If bleeding persists, embolization with coils, fat, microsphere/beads or thrombine is required. In our experience coils release through common microcatheter (i.e., Finecrossô, Terumo) is the safer and more feasible option. We prefer detachable coils that allow a more accurate placement.

"Balloon-Microcatheter Technique" (BMT) using microcatheters compatible with 6 Fr guiding catheters can be used for treatment of this type of perforation. The BMT consists of simultaneous advancement of a microcatheter over a parallel wire distal to the occluding balloon, in order to continue to operate through the microcatheter itself without deflating the occluding balloon ${ }^{[10]}$. This technique is able to accurately assess sealing of the perforation before and after the release of microcoils by tip injections from the jailed microcatheter, with no need of repeated deflations of the the proximal occluding balloon.

Collateral vessel perforation is a unique complication that may occur during retrograde CTO PCI. It is usually due to guidewires and/or devices advancement through the collateral or to balloon collateral dilation performed in order to facilitate retrograde devices passage. Progression to cardiac tamponade will depend on the location of the collateral vessel (i.e., septal vs. epicardial). Septal collateral perforation 

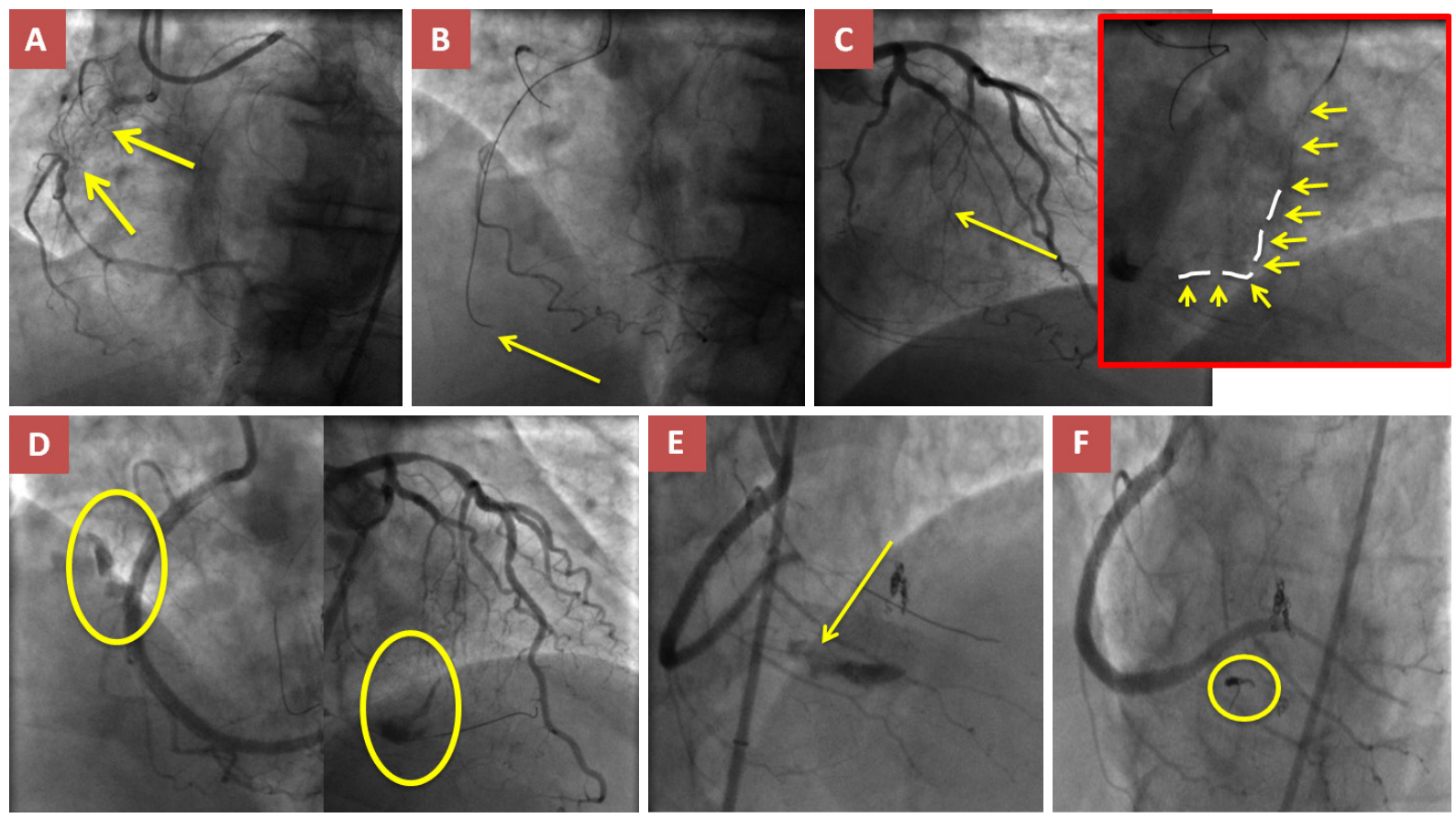

Figure 1. A: proximal RCA CTO; B: first antegrade approach was attempted but failed (guidewire outside of the vessel, yellow arrow); C: septal collateral was then chosen for retrograde attempt (see magnification in the red square); D: after reverse CART, guidewire externalization and stent implantation on RCA, perforation at level of mid RCA and septal branch were noticed (yellow circles); E: persistent extravasation and pericardial effusion increasing at echo despite covered stent implantation and coil release proximally to septal branch perforation; F: after coil release from retrograde source, no more contrast extravasation and stable pericardial effusion. CTO: chronic total occlusions; CART: controlled antegrade and retrograde tracking; RCA: right coronary artery

is typically considered a benign event because it commonly results in bleeding into interventricular septum. Septal wall hematomas that derive are usually asymptomatic, but can result in chest discomfort and rarely in heart block depending on its size and location ${ }^{[11,12]}$. Even more rarely, a septal wall hematoma can progress to a septal wall rupture ${ }^{[13]}$ or to obstruction of the ventricular cavity with subsequent cardiogenic shock. Unusual cases of right ventricular intramural hematoma following septal perforation are also reported ${ }^{[14]}$; they may be self-limiting or may result in hemodynamic compromise miming cardiac tamponade and then requiring emergency surgical evacuation. Septal vessel perforation can also take place into any cardiac chamber, including the coronary sinus: however, in this case, rarely any adverse clinical consequence occurs. In some cases septal perforations can be managed conservatively (especially when they have been caused by a guidewire) but embolization proximal to the perforation site should be considered for persisting extravasation. Anyway operators should keep in mind that in infrequent cases septal perforation may lead to pericardial effusion because these vessels can end in an epicardial course [Figure 1].

Epicardial collaterals perforation carries a high risk of pericardial effusion and rapid cardiac tamponade, due to their natural course. Moreover operators should remember that epicardial vessels receive blood flow from both antegrade and retrograde sources. Also in this case proximal balloon occlusion is the first maneuver to put in place. Then, unlike the cases above, the perforation should be approached both antegrade and retrograde. Usually embolization (i.e., coils) is the preferred treatment, both proximally and distally to the perforation site. In some cases perforation could be solved, on the antegrade side, only through a covered stent deployment in the MV interrupting blood supply to collateral. Obviously this approach presupposes that the CTO has been recanalized and the perforated vessel can be approached from both sides. At the end dual injection from both sides should be performed to confirm that there is no residual bleeding. If bleeding continues despite these measures, cardiac surgery may be required. 

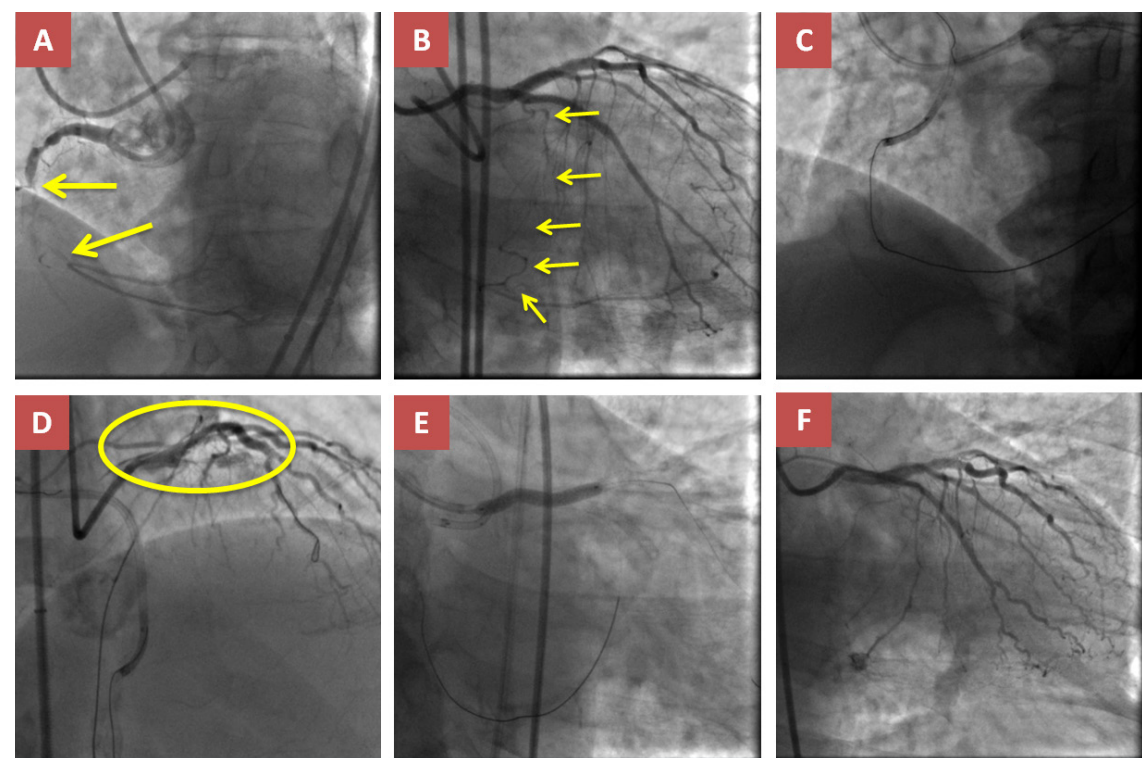

Figure 2. A:heavily calcified mid RCA CTO; B: epicardial collateral (yellow arrows) from LCx was navigated wih Sion Black wire; C: then CTO was crossed retrograde with Gaia 3 wire that re-entered in right guiding catheter but the microcatheter wasn't able to cross retrograde depite double trapping; D: clinical destabilization suddenly occurred with chest pain and hypotension: angiography showed LM dissection with LAD occlusion (circle); E: LM-LAD stenting after LAD wiring; F: final angiographic result: patency of LAD and LCx, aborted CTO PCI. CTO: chronic total occlusions; RCA: right coronary artery; LAD: left anterior descending; LCx: left circumflex; LM: left main

One last consideration should be made on epicardial perforations occurring in patients with prior coronary artery bypass graft. Typically, bleeding may be focal and contained in spaces due to prior adhesions and surgical scarring, without tamponade. Nevertheless local chamber compression (i.e., atria) with focal tamponade not amenable to pericardiocentesis and requiring surgical intervention, can still occur.

Despite new materials and techniques availability in the prevention and management of CPs, a crucial role is played by the identification of the clinical/angiographic predisposing factors. A recent study of Kinnaird et al.$^{[15]}$ investigates the principals features related to CPs during CTO PCI. Older age, female sex and previous PCI were found as main individual risk factors. Procedural factors associated with an increased risk of perforation were Crossboss/Stingray use, rotational or laser atherectomy, and microcatheter use.

\section{DONOR VESSEL INJURY (DISSECTION AND/OR OCCLUSION)}

Abrupt donor vessel injury represent a feared and unique complication occurring during retrograde CTO PCI. Its estimated incidence is $1.1 \%-1.8 \%{ }^{[16]}$. Leading mechanism of donor vessel injury are dissection (most common), thrombus formation/embolization, spasm or accidental air injection. Main effect of this complication is myocardial ischemia and its extension is related to the size of myocardium supplied. Thus, chest pain, electrocardiographic changes, hypotension and arrhythmias are common clinical features. Clinical and interventional management depends on the leading mechanism.

Dissection: usually retrograde CTO procedures require aggressive guiding catheters to gain the maximum support but at the expense of potential proximal donor vessel dissection. Furthermore specific attention must be paid in withdrawing equipment (wires, microcatheters), maneuver than can cause "deep intubation" and consequent dissection [Figure 2]. We recommend to place a protection guidewire along LAD at the beginning of the procedure, when retrograde approach from left coronary system is needed. 
If no placed before, intra-luminal guide-wire should be promptly advanced and, if correct position is not certain, a microcatheter should be used (laminar blood back-flow and eventually tip-injection can help to confirm correct position). Also IVUS could be used to avoid intimal injection and confirm correct wire position. Once intra-luminal wire position is confirmed, balloon inflation and stent implantation usually resolve the dissection.

If sub-intimal, the wire should be left in its position and a second guide-wire should be placed distally ("parallel wire"). Whether not possible, a re-entry technique must be considered.

Thrombus formation/embolization: during CTO PCI the activated clotted time (ACT) has to be controlled every $30 \mathrm{~min}$ at least, to ensure correct anticoagulation. It must be maintained above $350 \mathrm{~s}$. If ACT is low, correct with heparin infusion. In cases of heparin-resistance bivalirudin should be considered. When thrombosis occurs thrombus aspiration systems could be employed, followed by multiple balloon inflations but this strategy could lead to distal embolization. Gp2b/3a infusion should be also considered.

Spasm: guiding catheters and guidewires could induce an incremented vasoreactivity. In these cases intracoronary injection of vasodilators (es. Nitroglicerine, Adenosine, Nitroprusside) is recommended and usually resolve the spasm.

Accidental air injection: this is a rare eventuality that ideally should never happen. Meticulous bleed back from catheters and flushing after each device exchange should be practiced. Balloon rupture can also introduce air in the coronary arteries, though in small quantities. When occurring, air aspiration is crucial and could be combined to inotropic agents infusion. Is also possible to attempt bubbles breakdown with wires/balloons.

\section{EQUIPMENT LOSS OR ENTRAPMENT}

Equipment loss or entrapment during CTO PCI (guidewires, stents and other devices) usually represent a infrequent circumstance. Indeed it is a rarely reported complication so that its real incidence is unknown. The risk for this complication is related to lesion complexity, extent of calcifications, vessel tortuosity and techniques required for recanalization.

Knuckled wires have the potential of getting knotted. For this reason extreme caution should be paid when retrieving them in order to avoid entrapment. Guidewire fracture may also occur but rarely is associated with adverse clinical effects. Similarly microcatheters overtorquing could lead to entrapment.Balloon entrapment in highly calcified lesions and tortuous vessels a balloon could occur. In some cases balloons cannot be deflated for hypotube kinking. In both cases is crucial to remove the device avoiding distal ischemic damage. A controlled traction on the device avoiding the system fracture should be applied as first measure. If traction is not sufficient it would be necessary to advance a "guide extension catheter".

When stent loss occurs, retrieval should be attempted in all cases by distal balloon inflation and withdrawn together with the lost stent into the guide catheter. Not retrievable stent should be crushed against the vessel wall by multiple balloon inflations and stent deployment. Finally, adequate lesion preparation, is crucial to avoid stent loss during delivery attempt.

\section{CONTRAST-INDUCED NEPHROPATHY}

CTO PCI can be long-lasting procedures with administration of high volumes of contrast medium. Accurate patient selection should be performed in order to identify those at higher risk of CIN (incidence $3.4 \%$ ), carefully balancing the benefit-risk ratio of the procedure. Prevention of CIN is the first measure 
to be considered in all patients undergoing CTO interventions. Many prophylactic measures have been proposed to avoid kidney damage, however hydration with saline solution administration $(1 \mathrm{~mL} / \mathrm{kg}$ for $6 \mathrm{~h}$ before and $12 \mathrm{~h}$ after the procedure) has been proven to have the best outcomes.

During CTO PCI, repeated contrast injection should be avoided. Retrograde approach usually requires less contrast, likely due to use of "tip-injection". Similarly to non-CTO PCI, IVUS employment can contribute to reduce contrast amount in several ways (IVUS-guided antegrade puncture, vessel diameter and disease extension evaluation).

\section{VASCULAR ACCESS COMPLICATIONS}

CTO PCI usually requires dual arterial access and larger sheath diameter, compared to non-CTO PCI. Both conditions increase the probability of vascular access complications with reported rate of $0.5 \%-1.5 \%{ }^{[17]}$. Compared with femoral access, radial approach is associated with lower adverse cardiac events and major vascular complications rates across the entire spectrum of patients with stable or unstable CAD ${ }^{[18]}$. Moreover, the development of thin-walled sheaths (e.g., 7 in 6 French Glidesheath Slender ${ }^{\mathrm{TM}}$, Terumo) and sheathless techniques made feasible complex PCIs requiring 7 French catheters also from the radial access. Indeed, even in CTO PCI, a fully transradial approach has been proven to be safe with a high rate of success and low complications incidence ${ }^{[19]}$. On the other hand, due to technical aspects (e.g., multiple devices housing in the same catheter), 8 French catheters may be needed and in these cases femoral access is the most practical way. Furthermore, depending on anatomical characteristics, when strong back up support is desirable, $45 \mathrm{~cm}$ long femoral sheaths could provide it. For all these reasons vascular access choice is left to the operator's discretion.

\section{RADIATION SKIN INJURY}

Radiation skin injury is of particular concern in patients undergoing CTO PCI, as long fluoroscopy and cine-angiography exposure may be required to cross and treat the lesion. However radiation injury incidence is low $(<0.01 \%)^{[5]}$, but the data are under-reported in literature. Tricks to minimize radiation exposure are reducing frame rate of fluoroscopy and cine; another ploy is the use of stored fluoroscopy instead of angiography. Newer angiographic devices with low-dose settings could also reduce patient radiation exposure.

\section{CONCLUSION}

CTOs represent the most technically challenging lesions that interventional cardiologists face in everyday practice. However, due to newer dedicated devices and improved techniques, the rate of successful CTOs recanalization is increasing whereas the rate of complications is reducing. Nevertheless peri-procedural complications incidence, particularly in retrograde approach, is still higher than in non-CTO PCI. Operators approaching to CTO PCI should also be aware of the unique set of complications associated with CTOs recanalization. Consciousness of the potential specific CTO PCI complications is the first step to prevent and solve them. Then the knowledge of techniques and equipment available for complications management, combined with operators' experience, will contribute to safe percutaneous treatment of CTOs.

\section{DECLARATIONS}

\section{Authors' contributions}

Searched for literature data and wrote the paper: Colombo F, Bernardi A

Conceived the structure of the chapter and reviewed the manuscript: Garbo R 


\section{Availability of data and materials}

All the literature data (articles, case reports and reviews) supporting this book chapter were collected from Pubmed and are open source available.

\section{Financial support and sponsorship}

None.

\section{Conflicts of interest}

All authors declared that there are no conflicts of interest.

\section{Ethical approval and consent to participate}

Not applicable.

\section{Consent for publication}

Not applicable.

\section{Copyright}

(c) The Author(s) 2019.

\section{REFERENCES}

1. Patel SM, Menon RV, Burke MN, Jaffer FA, Yeh RW, et al. Current Perspectives and Practices on Chronic Total Occlusion Percutaneous Coronary Interventions. J Invasive Cardiol 2018;30:43-50.

2. Sapontis J, Salisbury AC, Yeh RW, Cohen DJ, Hirai T, et al. Early Procedural and Health Status Outcomes After Chronic Total Occlusion Angioplasty: A Report From the OPEN-CTO Registry (Outcomes, Patient Health Status, and Efficiency in Chronic Total Occlusion Hybrid Procedures). JACC Cardiovasc Interv 2017;10:1523-34.

3. Galassi AR, Tomasello SD, Reifart N, Werner GS, Sianos G, et al. In-hospital outcomes of percutaneous coronary intervention in patients with chronic total occlusion: insights from the ERCTO (European Registry of Chronic Total Occlusion) registry. EuroIntervention 2011;7:472-9.

4. Shimony A, Joseph L, Mottillo S, Eisenberg MJ. Coronary artery perforation during percutaneous coronary intervention: a systematic review and meta-analysis. Can J Cardiol 2011;27:843-50.

5. Patel VG, Brayton KM, Tamayo A, Mogabgab O, Michael TT, et al. Angiographic success and procedural complications in patients undergoing percutaneous coronary chronic total occlusion interventions: a weighted meta-analysis of 18,061 patients from 65 studies. JACC Cardiovasc Interv 2013;6:128-36.

6. Danek BA, Karatasakis A, Tajti P, Sandoval Y, Karmpaliotis D, et al. Incidence, Treatment, and Outcomes of Coronary Perforation During Chronic Total Occlusion Percutaneous Coronary Intervention. Am J Cardiol 2017;120:1285-92.

7. Fiocca L, Cereda AF, Bernelli C, Canova PA, Serino F, et al. Autologous blood reinfusion during iatrogenic acute hemorrhagic cardiac tamponade: Safety and feasibility in a cohort of 30 patients. Catheter Cardiovasc Interv 2019;93:E56-E62.

8. Ellis SG, Ajluni S, Arnold AZ, Popma JJ, Bittl JA, et al. Increased coronary perforation in the new device era. Incidence, classification, management, and outcome. Circulation 1994;90:2725-30.

9. Brilakis ES. Manual of Coronary Chronic Total Occlusion Interventions. A Step-By-Step Approach. Academic Press. 2014; DOI: 10.1016/B978-0-12-420129-3.00019-2.

10. Garbo R, Oreglia JA, Gasparini GL. The Balloon-Microcatheter Technique for Treatment of Coronary Artery Perforation. Catheter Cardiovasc Interv 2017;89:E75-E83.

11. Joyal D, Thompson CA, Grantham JA, Buller CE, Rinfret S. The retrograde technique for recanalization of chronic total occlusions: a step-by-step approach. JACC Cardiovasc Interv 2012;5:1-11.

12. Lin TH, Wu DK, Su HM, Chu CS, Voon WC, et al. Septum hematoma: a complication of retrograde wiring in chronic total occlusion. Int J Cardiol 2006;113:e64-6.

13. Fairley SL, Donnelly PM, Hanratty CG, Walsh SJ. Interventricular septal hematoma and ventricular septal defect after retrograde intervention for a chronic total occlusion of a left anterior descending coronary artery. Circulation 2010;122:e518-21.

14. Ghobrial MSA, Egred M. Right Ventricular Wall Hematoma Following Angioplasty to Right Coronary Artery Occlusion. J Invasive Cardiol 2019;31:E66.

15. Kinnaird T, Anderson R, Ossei-Gerning N, Cockburn J, Sirker A, et al. Legacy Effect of Coronary Perforation Complicating Percutaneous Coronary Intervention for Chronic Total Occlusive Disease: An Analysis of 26807 Cases From the British Cardiovascular Intervention Society Database. Circ Cardiovasc Interv 2017;10. DOI: 10.1161/CIRCINTERVENTIONS.116.004642.

16. Tajti P, Burke MN, Karmpaliotis D, Alaswad K, Jaffer FA, et al. Prevalence and Outcomes of Percutaneous Coronary Interventions for 
Ostial Chronic Total Occlusions: Insights From a Multicenter Chronic Total Occlusion Registry. Can J Cardiol 2018;34:1264-74.

17. Kinnaird T, Anderson R, Ossei-Gerning N, Gallagher S, Large A, et al. Vascular Access Site and Outcomes Among 26,807 Chronic Total Coronary Occlusion Angioplasty Cases From the British Cardiovascular Interventions Society National Database. JACC Cardiovasc Interv 2017;10:635-44.

18. Ferrante G, Rao SV, Jüni P, Da Costa BR, Reimers B, et al. Radial Versus Femoral Access for Coronary Interventions Across the Entire Spectrum of Patients With Coronary Artery Disease: A Meta-Analysis of Randomized Trials. JACC Cardiovasc Interv 2016;9:1419-34.

19. Bakker EJ, Maeremans J, Zivelonghi C, Faurie B, Avran A, et al. Fully Transradial Versus Transfemoral Approach for Percutaneous Intervention of Coronary Chronic Total Occlusions Applying the Hybrid AlgorithmInsights From RECHARGE Registry. Circ Cardiovasc Interv 2017;10. DOI: 10.1161/CIRCINTERVENTIONS.117.005255. 